\title{
Soro de leite como causa de alta mortalidade de bovinos $^{1}$
}

\author{
Aldo Gava ${ }^{2 *}$, Franciéli A. Molossi², Nathalia S. Wicpolt'², Daiane Ogliari², \\ Thalita C. Cardoso 2 , Sandra D. Traverso ${ }^{2}$ e Claudia S. Wisser ${ }^{2}$
}

\begin{abstract}
Gava A., Molossi F.A., Wicpolt N.S., Ogliari D., Cardoso T.C., Traverso S.D. \& Wisser C.S. 2018. [Whey as cause of high mortality in cattle.] Soro de leite como causa de alta mortalidade de bovinos. Pesquisa Veterinária Brasileira 38(4):620-623. Laboratório de Patologia Animal, Departamento de Medicina Veterinária, Centro de Ciências Agroveterinárias, Universidade do Estado de Santa Catarina, Av. Luiz de Camões 2090, Lages, SC 88520-000, Brazil. E-mail: aldo.gava@udesc.br

This study reports the epidemiological data, clinical signs and lesions produced in the rumen by ingestion of fermented whey. 160 cattle have become ill, including cows, heifers and calves, 87 of them died. One day after being given 6000 liters of whey, the cattle showed loss of appetite, staggering gait, endophthalmitis, diarrhea with gray coloration and malodorous, and they lay down and raised frequently. Necropsy findings were consistent in the rumen and were characterized by congestion of vessels of serous and marked distention of the wall, allowing seeing the internal content, which was liquid, greyish and fetid. The mucosa was reddish with junction of the buds forming small masses of firm consistency. In the abomasum the mucosa was dark red, fuzzy. Histological lesions in the rumen consisted of vacuolar degeneration with formation of vesicles, multifocal necrosis accompanied by neutrophilic infiltrate, sometimes with fibrin deposition, besides thickening of the keratinized mucosa layer.
\end{abstract}

INDEX TERMS: Acidosis, cattle, whey.

RESUMO.- Descrevem-se os dados epidemiológicos, sinais clínicos e lesões produzidas no rúmen pela ingestão de soro de leite fermentado. Adoeceram 160 bovinos incluindo vacas, novilhas ebezerras, dos quais 87 morreram. Um dia após terem sido fornecidos 6.000 litros de soro de leite, os bovinos manifestaram perda de apetite, andar cambaleante, endoftalmia, diarreia de coloração cinza e fétida e deitavam e levantavam com frequência. Os achados de necropsia foram consistentes no rúmen e se caracterizaram por congestão dos vasos da serosa e distensão acentuada da parede, permitindo observar o conteúdo interno, que era líquido, acinzentado e fétido. A mucosa tinha coloração avermelhada com junção das papilas formando pequenas massas de consistência firme. No abomaso a mucosa era vermelho escura, difusa. As lesões histológicas no rúmen consistiram de degeneração vacuolar com formação de vesículas, necrose multifocal acompanhada de infiltrado neutrofílico, as vezes com deposição de fibrina, além de espessamento da camada queratinizada da mucosa.

TERMOS DE INDEXAÇÃO: Acidose, bovino, soro de leite.

\footnotetext{
${ }^{1}$ Recebido em 22 de dezembro de 2016.

Aceito para publicação em 11 de abril de 2017.

${ }^{2}$ Laboratório de Patologia Animal, Departamento de Medicina Veterinária, Centro de Ciências Agroveterinárias, Universidade do Estado de Santa Catarina (CAV/UDESC), Av. Luiz de Camões 2090, Bairro Conta Dinheiro, Lages, SC 88520-000, Brasil. *Autor para correspondência: aldo.gava@udesc.br
}

\section{INTRODUÇÃO}

O soro de leite é um dos subprodutos resultantes da fabricação de queijo, cuja composição vem sendo amplamente estudada no Brasil, a fim de identificar alternativas para aproveitamento adequado do mesmo, devido à sua alta qualidade nutricional, do seu volume e de seu poder poluente (Susmel et al. 1995, Camargo et al. 2000, FEAM 2003, Fontes et al. 2006, David et al. 2010, Paula et al. 2011, Morril et al. 2012). Ele é constituído basicamente de $83,57 \%$ de umidade, $6,43 \%$ de matéria seca, $4,25 \%$ de lactose, $1,25 \%$ de proteína bruta, $0,33 \%$ de gordura, $0,60 \%$ de sais minerais e pH de 6,34 (Barbosa et al. 2010). No Brasil, a produção de bebidas lácteas é uma das principais opções de aproveitamento do soro do leite (Capitani et al. 2005). Suas aplicações industriais são diversas na produção de alimentos ou, indústria farma/nutracêutica (panificação, sorvetes, misturas lácteas ou leites modificados, chocolates, suplementos esportivos e nutricionais, fórmulas infantis e outros) (Homem 2004).

A utilização do soro do leite pode entrar na alimentação animal de ruminantes, suínos e aves e na alimentação humana sob as formas líquida, condensada, ou em pó (Mizubuti 1994). O soro pode ser considerado como uma fonte de energia na alimentação dos animais devido ao seu alto conteúdo em lactose, porém os ruminantes são capazes de digerir quantidade limitada de lactose no rúmen e intestinos (Schingoethe 1976). 
Em geral, o soro do leite pode ser utilizado na dieta de bovinos em níveis de até $44 \%$ de matéria seca, sem causar distúrbios digestivos (Susmel et al. 1995). Entretanto, alimentos ricos em carboidratos altamente fermentáveis (lactose e/ou glicose, amido, sacarose), quando ingeridos em excesso por bovinos e sem adaptação prévia da flora ruminal, podem causar acidose lática ruminal (Radostits et al. 2002, Smith 2006). Estes carboidratos aceleram o processo fermentativo ruminal, ocasionando supercrescimento dos estreptococos que por sua vez produzem grande quantidade de ácido lático com consequente diminuição do $\mathrm{pH}$ (Radostits et al. 2002). 0 soro de leite líquido fermenta com facilidade, o que resulta em aumento da quantidade de ácido lático ingerido pelos animais, contribuindo para a queda do $\mathrm{pH}$ do rúmen (Schingoethe et al. 1979). O aumento na concentração de ácido lático ruminal aumenta a pressão osmótica do material ingerido resultando na passagem de água e eletrólitos do sangue para o interior do rúmen, causando desidratação e eventualmente colapso circulatório. Além disso, o excesso de ácido lático desequilibra a microbiota ruminal normal e tem ação corrosiva para a mucosa do rúmen o que provoca a perda do epitélio desse órgão (McGavin \& Zachary 2013).

Clinicamente os bovinos apresentam anorexia, depressão, desidratação, estase ruminal, diarreia intensa, fraqueza e ataxia (Radostits et al. 2002). Os achados de necropsia são caracterizados por conteúdo ruminal e intestinal aquoso e ácido, mucosa das papilas ruminais marrom que se desprende com facilidade, e na microscopia há degeneração hidrópica e necrose de coagulação do epitélio ruminal, seguida de infiltrado de neutrófilos (McGavin \& Zachary 2013).

0 objetivo do presente trabalho é relatar um surto de acidose láctica ruminal em bovinos, causado pela ingestão de soro de leite em quantidade e condições inadequadas para o consumo dos animais.

\section{MATERIAL E MÉTODOS}

Os dados epidemiológicos e sinais clínicos foram obtidos através de visita a uma propriedade localizada no município de Treze Tílias, Santa Catarina. Três vacas e três novilhas foram necropsiadas e fragmentos de rim, pulmão, retículo, rúmen, intestinos, músculo, coração, omaso, abomaso, fígado, glândula parótida, linfonodos, baço e sistema nervoso central foram coletados e fixados em formalina à $10 \%$ e processados rotineiramente para avaliação histológica.

\section{RESULTADOS}

A propriedade possuía 160 bovinos, da raça holandesa, incluindo vacas, novilhas e bezerras. Durante o dia, os bovinos eram mantidos em pastagem e a noite em piquete de aproximadamente um hectare onde recebiam soro de leite adicionado à silagem de milho. Devido à escassez de alimentos, os bovinos passaram a permanecer fechados em uma pequena área sem pastagem e alimentados somente com silagem de milho. Três dias após, foram fornecidos 6.000 litros de soro do fundo do tanque de armazenamento contendo grande quantidade de gordura, o qual foi misturado à silagem de milho. No dia seguinte os bovinos manifestaram perda de apetite, andar cambaleante, endoftalmia, diarreia de coloração cinza e fétida, deitavam e levantavam com frequência. No decorrer de cinco dias após a administração do soro, morreram 87 bovinos. Os achados de necropsia dos seis bovinos necropsiados consistiram de congestão dos vasos da serosa e distensão acentuada da parede do rúmen (Fig.1). A mucosa tinha coloração avermelhada com junção das papilas formando pequenas massas de consistência firme e o conteúdo era líquido, acinzentado e fétido (Fig.2). No abomaso a mucosa era vermelho escura, difusa. As alterações histológicas foram relevantes no rúmen e caracterizaram-se por degeneração vacuolar das células epiteliais, com formação de vesículas, por vezes, necrose multifocal acompanhada de infiltrado neutrofílico, às vezes com deposição de fibrina, além de espessamento da camada queratinizada da mucosa (Fig.3).

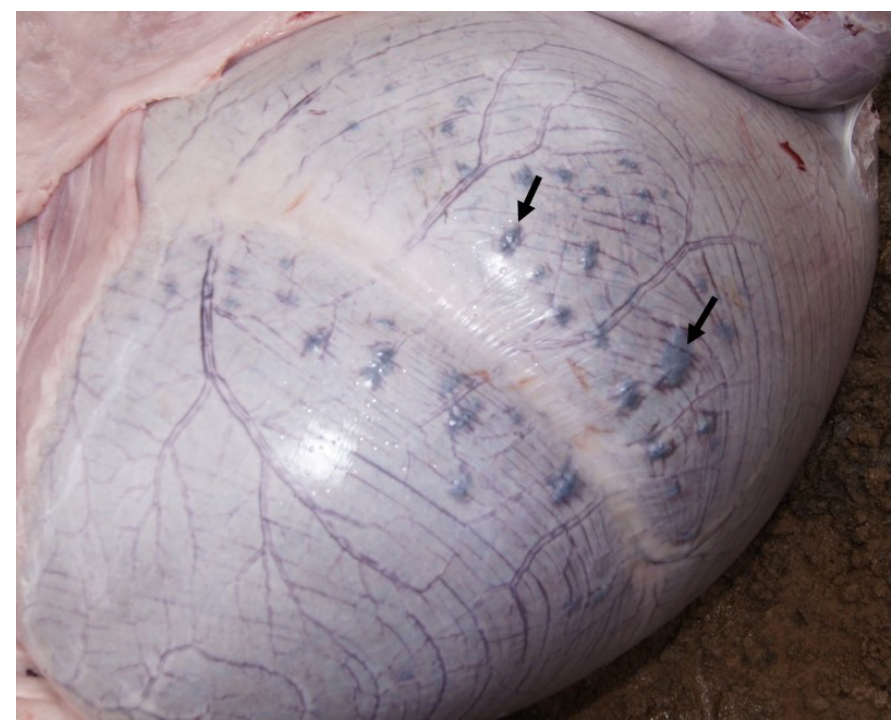

Fig.1. Rúmen bovino, acidose por soro de leite. Congestão de vasos da serosa, distensão da parede do rúmen e junção de grupos de papilas vistas externamente (setas).

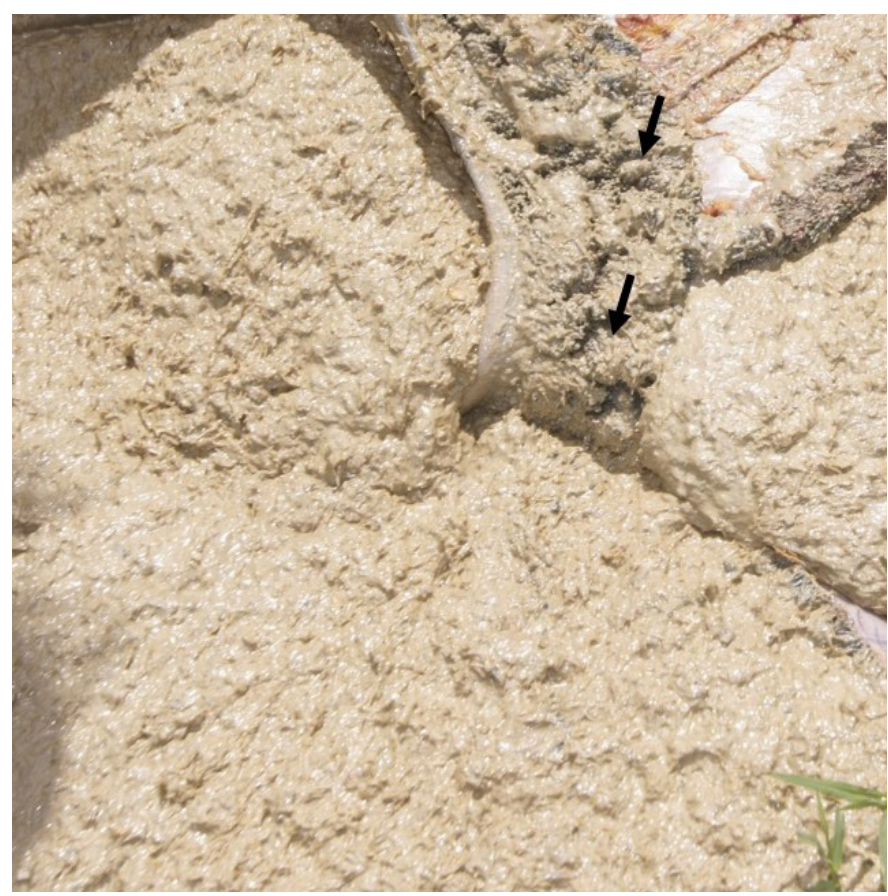

Fig.2. Rúmen bovino, acidose por soro de leite. Conteúdo ruminal após a ingestão de soro de leite e junção de grupos de papilas na mucosa (setas). 


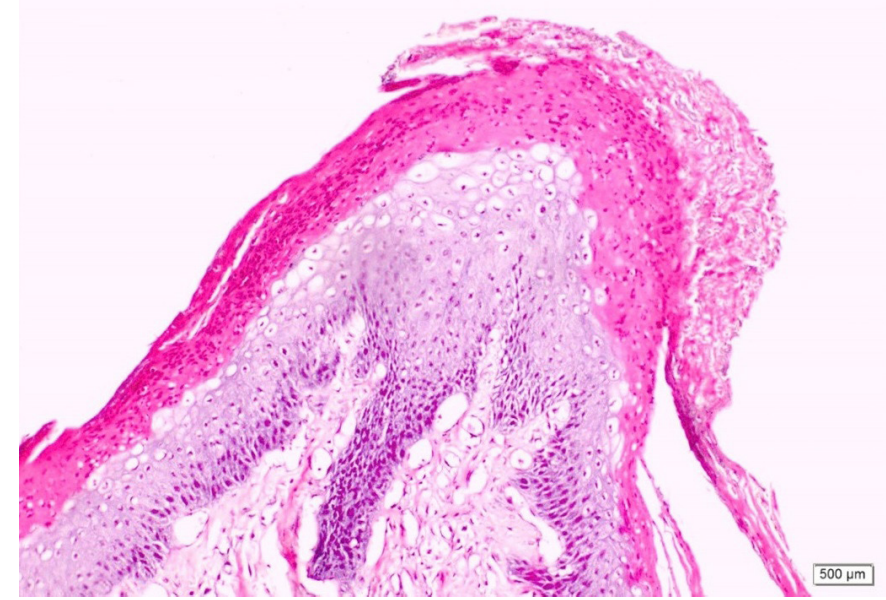

Fig.3. Rúmen bovino, acidose por soro de leite. Necrose da capa queratinizada e infiltração de neutrófilos acompanhado de degeneração vacuolar de células epiteliais. HE, obj.10x.

\section{DISCUSSÃO}

O diagnóstico de acidose ruminal baseou-se nos dados epidemiológicos, sinais clínicos, achados macroscópicos e microscópicos e caracterizou-se pela ingestão de grande quantidade de soro de leite fermentado sem adaptação prévia da microbiota ruminal.

Casos de timpanismo em novilhos alimentados com soro de leite foram observados por Lynch et al. (1975), todavia não existe comprovação científica que a administração desta quantidade ou quantidades superiores de soro de leite líquido fermentado e com gordura possa causar a morte de bovinos.

Os principais sinais clínicos associados à doença, foram semelhantes aos relatados para a acidose, por Radostits et al. (2002), e incluem perda de apetite, ataxia, endoftalmia e diarreia. Os achados de necropsia caracterizaram-se principalmente pela distensão da parede ruminal, conteúdo líquido e fétido. Ao exame histológico, as lesões foram marcantes no rúmen e estão de acordo com as descritas para acidose ruminal por McGavin \& Zachary (2013).

A morte dos animais devido a ingestão de soro de leite pode estar relacionada a vários fatores. 0 primeiro deles é a quantidade de soro oferecida abruptamente aos animais. Segundo David et al. (2010), o soro deve ser introduzido gradativamente na dieta, para que ocorra o ajuste da microbiota ruminal à nova fonte de energia, principalmente devido a seus elevados teores de lactose. Outro fator que pode ter contribuído com a morte dos animais foi a utilização do resíduo de soro do tanque de armazenamento, o qual estava associado a grande quantidade de gordura. 0 impacto de teores elevados de gordura na dieta de bovinos ainda está longe de ser elucidado. No entanto, evidências limitadas sugerem que as gorduras com altas proporções de ácidos graxos livres podem inibir a biohidrogenação ruminal (Noble et al. 1974). Além disso, dietas com alto teor de carboidratos não fibrosos influenciam negativamente a biohidrogenação (Oelker et al. 2009). Assim, se a biohidrogenção é inibida, ocorre a toxidez/desequilíbrio dos microrganismos ruminais, podendo contribuir com a queda no $\mathrm{pH}$ do rúmen. Segundo Zinn \& Jorquera (2007), 3\% de suplementação de gordura já é suficiente para afetar negativamente o desempenho dos animais não adaptados e isto não está relacionado ao valor de energia da gordura, mas sim, com a palatabilidade que afeta o consumo de alimentos dos animais.

Os bovinos do presente estudo ingeriram soro de leite juntamente com a silagem de milho. De acordo com Susmel et al. (1995), o soro de leite modifica padrões de fermentação ruminal causando uma rápida queda no $\mathrm{pH}$ ruminal. Algumas silagens contêm tanto carboidratos como ácido lático em grandes quantidades e acabam por introduzir mais ácido à ingestão e fermentação (Smith 2006). Adicionalmente, segundo Schingoethe et al. (1979), o soro de leite líquido fermenta rapidamente e produz ácido lático.

A escassez de alimento volumoso também pode ter contribuído para a ocorrência da doença, uma vez que, menores quantidades de fibra estrutural induzem menor salivação e menos ruminação, portanto, os tampões salivares diminuem, o que contribui com a queda no $\mathrm{pH}$ ruminal (Smith 2006). Além disso, a redução do $\mathrm{pH}$ por excesso de carboidratos solúveis diminui a digestibilidade das fibras (Oelker et al. 2009), contribuindo para o surgimento do quadro clínico-lesional.

As lesões ruminais decorrentes da ingestão de soro de leite devem ser diferenciadas de outras doenças que se apresentem na forma de surto, com lesões principalmente no tubo digestivo, dentre elas, intoxicação por Eupatorium tremulum (Lucioli et al. 2007); Baccharis coridifolia (Tokarnia \& Döbereiner 1975, Barros 1993, Varaschin et al. 1998, Rissi et al. 2005); Baccharis megapotamica var. weirii e var. megapotamica (Tokarnia et al. 1992) e Baccharidastrum triplinervium (Langohr et al. 2005). Inclui-se ainda como diagnóstico diferencial, a acidose ruminal causada pela ingestão de carboidratos altamente fermentáveis, como lactose, glicose, amido e sacarose, pois provocam lesões semelhantes nos bovinos. 0 tratamento descrito para acidose ruminal e sistêmica, deve ser realizado com uso de agentes alcalinizantes, fluidoterapia, restauração da motilidade dos pré-estômagos com auxílio de feno (Radostits et al. 2002) e uso de antibióticos ionóforos (Nagaraja et al.1981, 1982, Erickson et al. 2003, Oelker et al. 2009). Além disso, para animais de até $200 \mathrm{Kg}$ de peso vivo, o consumo deve ser limitado para 10 a 15 litros/soro/animal/dia. Para bovinos com mais de $200 \mathrm{Kg}$ o soro deve ser fornecido até no máximo $30 \%$ do consumo total de matéria seca. 0 soro de leite fresco deve ser fornecido separadamente, livre à escolha dos animais ou em rações completas. Os animais precisam de um período de adaptação de aproximadamente duas semanas com aumento gradativo da quantidade oferecida para evitar timpanismo e diarreia (Anderson et al. 1974, Lizieire \& Campos 2006).

\section{CONCLUSÃO}

O oferecimento abrupto de grande quantidade de soro de leite fermentado e rico em gordura, associado à restrição de alimentos volumosos provocaram acidose ruminal em bovinos e podem ser responsabilizados pela alta mortalidade.

\section{REFERÊNCIAS}

Anderson M.J., Lamb R.C., Mickelsen C.H. \& Wiscombe R.L. 1974. Feeding liquid whey to dairy cattle. J. Dairy Sci. 57(10):1206-1210. http://dx.doi. org/10.3168/jds.S0022-0302(74)85038-1. 
Barbosa A.S., Florentino E.R., Florêncio I.M. \& Araújo A.S. 2010. Utilização do soro como substrato para produção de aguardente: estudo cinético da produção de etanol. Revta Verde 5(1):7-25.

Barros C.S.L. 1993. Intoxicação por Baccharis coridifolia, p.159-169. In: Riet-Correa F., Méndez M.C. \& Schild A.L. (Eds), Intoxicações por Plantas e Micotoxicoses em Animais Domésticos. Agropecuária Hemisfério Sul S.R.L., Montevideo.

Camargo D.S., Alves G., Garcia S. \& Mizubuti I.Y. 2000. Bebida fermentada à base de soro de leite e isolado protéico de soja. Semina, Ciênc. Agrárias 21(1):45-51. http://dx.doi.org/10.5433/1679-0359.2000v21n1p45.

Capitani C.D., Pacheco M.T.B., Gumerato H.F., Vitali A. \& Schmidt F.L. 2005. Recuperação de proteínas do soro de leite por meio de conservação com polissacarídeo. Pesq. Agropec. Bras. 40(11):1123-1128. http://dx.doi. org/10.1590/S0100-204X2005001100010.

David F.M., Collao-Saenz E.A., Pérez J.R.O., Castro A.L.A., Resende H.R.A. \& Landim A.V. 2010. Efeito da adição de soro de leite sobre a digestibilidade aparente e os parâmetros sanguíneos de vacas secas. Arq. Bras. Med. Vet. Zootec. 62(5):1183-1190. http://dx.doi.org/10.1590/S0102-09352010000500022.

Erickson G.E., Milton C.T., Fanning K.C., Cooper R.J., Swingle R.S., Parrott J.C., Vogel G. \& Klopfenstein T.J. 2003. Interaction between bunk management and monensin concentration on finishing performance, feeding behavior, and ruminal metabolism during an acidosis challenge with feedlot cattle. J. Anim. Sci. 81(11):2869-2879. http://dx.doi.org/10.2527/2003.81112869x. PMid:14601891.

FEAM 2003. Lei Federal no 14.940, de 29 de dezembro de 2003. Taxa de Controle e Fiscalização Ambiental (TFAMG) das atividades potencialmente poluidoras e utilizadoras de recursos naturais. Diário Oficial da República Federativa do Brasil, Brasília, DF.

Fontes F.A.P.V., Coelho S.G., Lana A.M.Q., Costa T.C., Carvalho A.U., Ferreira M.I.C., Saturnino H.M., Reis R.B. \& Serrano A.L. 2006. Desempenho de bezerros alimentados com dietas líquidas à base de leite integral ou soro de leite. Arq. Bras. Med. Vet. Zootec. 58(2):212-219. http://dx.doi.org/10.1590/ S0102-09352006000200010.

Homem G.R. 2004. Avaliação técnico-econômica e análise locacional de unidade processadora de soro de queijo em Minas Gerais. Tese de Doutorado em Ciência e Tecnologia de Alimentos, Universidade Federal de Viçosa, Viçosa. 253p.

Langohr I.M., Gava A. \& Barros C.S.L. 2005. Intoxicação por Baccharidastrum triplinervuim (Asteraceae) em bovinos. Pesq. Vet. Bras. 25(4):235-238. http://dx.doi.org/10.1590/S0100-736X2005000400009.

Lizieire R.S. \& Campos O.F. 2006. Soro de Queijo "in natura" na Alimentação do Gado de Leite. $2^{\text {a }}$ ed. Embrapa Gado de Leite, Juiz de Fora.

Lucioli J., Furlan F.H., Mezaroba S., Traverso S.D. \& Gava A. 2007. Intoxicação espontânea e experimental por Eupatorium tremulum (Asteraceae) em bovinos. Pesq. Vet. Bras. 27(10):442-445. http://dx.doi.org/10.1590/ S0100-736X2007001000010.

Lynch G.P., McDonough F.E., Rough D.K., Smith D.F. \& Gordon C.H. 1975. Growth and carcass evaluation of Holstein steers fed liquid acid whey. J. Dairy Sci. 58(11):1688-1694. http://dx.doi.org/10.3168/jds.S00220302(75)84769-2. PMid:1194469.

McGavin M.D. \& Zachary J.F. 2013. Sistema alimentar, peritônio, omento, mesentério e cavidade peritonial. In: Ibid. (Eds), Bases da Patologia em Veterinária. $2^{2}$ ed. Elsevier, Rio de Janeiro. p.345-346.

Mizubuti I.Y. 1994. Soro de leite: composição, processamento e utilização na alimentação. Semina, Ciênc. Agrárias 15(1):80-94.
Morril W.B.B., Rolim M.M., Neto E.B., Pedrosa E.M.R., Oliveira V.S. \& Almeida G.L.P. 2012. Produção e nutrientes minerais de milheto forrageiro e sorgo sudão adubado com soro de leite. Revta Bras. Eng. Agríc. Ambient. 16(2):182-188.

Nagaraja T.G., Avery T.B., Bartley E.E., Galitzer S.J. \& Dayton A.D. 1981 Prevention of lactic acidosis in cattle by lasalocid or monensin. J. Anim. Sci. 53(1):206-216. http://dx.doi.org/10.2527/jas1981.531206x. PMid:7319937.

Nagaraja T.G., Avery T.B., Bartley E.E., Roof S.K. \& Dayton A.D. 1982. Effect of lasalocid, monensin or thiopectin on lactic acidosis in cattle. J. Anim. Sci. 54(3):649-658. http://dx.doi.org/10.2527/jas1982.543649x. PMid:7085521.

Noble R.C., Moore J.H. \& Harfoot C.G. 1974. Observations of the pattern of biohydrogenation of esterified and unesterified linoleic acid in the rumen. Brit. J. Nutr. 31(1):99-108. http://dx.doi.org/10.1079/BJN19740012. PMid:4810360.

Oelker E.R., Reveneau C.E. \& Firkins J.L. 2009. Interaction of molasses and monensin in alfalfa hay- or corn silage-based diets on rumen fermentation, total tract digestibility, and milk production by Holstein cows. J. Dairy Sci. 92(1):270-285. http://dx.doi.org/10.3168/jds.2008-1432. PMid:19109286.

Paula L., Rolim M.M., Bezerra Neto E., Soares T.M., Pedrosa E.M.R. \& Silva E.F.F. 2011. Crescimento e nutrição mineral de milho forrageiro em cultivo hidropônico com soro de leite bovino. Revta Bras. Eng. Agríc. Ambient. 15(9):931-939. http://dx.doi.org/10.1590/S1415-43662011000900009.

Radostits O.M., Gay C.C., Blood D.C. \& Hinchcliff K.W. 2002. Doenças do trato alimentar. In: Radostits O. M., Gay C.C., Blood D.C. \& Hinchcliff K.W. (Eds), Clínica Veterinária. 9a ed. Guanabara Koogan, Rio de Janeiro p.257-264.

Rissi D.R., Rech R.R., Fighera R.A., Cagnini D.Q., Kommers G.D. \& Barros C.S.L. 2005. Intoxicação espontânea por Baccharis coridifolia em bovinos. Pesq. Vet. Bras. 25(2):111-114. http://dx.doi.org/10.1590/S0100736X2005000200008

Schingoethe D.J. 1976. Whey utilization in animal feeding: a sumary and evaluation. J. Dairy Sci. 59(2):556-570. http://dx.doi.org/10.3168/jds. S0022-0302(76)84240-3.

Schingoethe D.J., Skyberg E.W. \& Bailey R.W. 1979. Digestibility, mineral balance, and rumen fermentation by steers of rations containing large amounts of lactose or dried whey. J. Dairy Sci. 63(5):762-774. http:// dx.doi.org/10.3168/jds.S0022-0302(80)83005-0.

Smith B.P. 2006. Enfermidades do trato alimentar. In: Murray J.M. \& Smith B.P. (Eds), Medicina Interna de Grandes Animais. 3aㅡ ed. Manole, Barueri. p.731-733.

Susmel P., Spanghero M., Mills C.R. \& Stefanon B. 1995. Rumen fermentation characteristics and digestibility of cattle diets containing different whey: maize ratios. Anim. Feed Sci. Technol. 53(1):81-89. http://dx.doi org/10.1016/0377-8401(95)00724-2.

Tokarnia C.H. \& Döbereiner J. 1975. Intoxicação experimental em bovinos por "mio-mio" (Baccharis coridifolia). Pesq. Vet. Bras. 10:79-97.

Tokarnia C.H., Peixoto P.V., Gava A. \& Barros C.S.L. 1992. Intoxicação experimental por Baccharis megapotamica var. megapotamica e var. weirii (Compositae) em bovinos. Pesq. Vet. Bras. 12(1/2):19-31.

Varaschin M.S., Barros C.S.L. \& Jarvis B.B. 1998. Intoxicação experimental por Baccharis coridifolia (Compositae) em bovinos. Pesq. Vet. Bras. 18(2):6974. http://dx.doi.org/10.1590/S0100-736X1998000200006.

Zinn R.A. \& Jorquera A.P. 2007. Feed value of supplemental fats used in feedlot cattle diets. Vet. Clin. N. Am., Food Anim. Pract. 23(2):247-268. http:// dx.doi.org/10.1016/j.cvfa.2007.03.003. PMid:17606149. 